VOL. $3(1970), 139$

\title{
Abstracts of Australasian $\mathrm{Ph} \mathrm{D}$ theses
}

\section{On the Mathieu group $M_{23}$}

\author{
Nita Bryce
}

The main theorem of the thesis is:

MAIN THEOREM. Let $G$ be a finite non-abelian simple group of order $10,200,960$. Then $G$ is isomorphic to $M_{23}$.

We have thus affirmed that all the Mathieu groups are uniquely determined by their order. The unique determination by order of the Mathieu groups $M_{12}, M_{24}, M_{11}$ and $M_{22}$ has been proved by R.G. Stanton (Ph.D. thesis, University of Toronto 1948) and D.L. Parrott (Ph.D. thesis, Moriash University 1969).

Using the Sylow theorems and block theoretic arguments as developed primarily by $R$. Brauer, the structure of the centralizer of an involution in the centre of a Sylow 2-subgroup in $G$ is found. A result of $Z$. Janko which characterizes the Mathieu group $M_{23}$ in terms of the centralizer of a central involution is used to complete the proof of the theorem.

Received 4 April 1970. Thesis submitted to Monash University, May 1969. Degree approved, October 1969. Supervisor: Professor Z. Janko. 139 